\title{
$4 \quad$ Measuring the temporal quality of a biodiversity database
}

Les G Underhill

7

8 Department of Biological Sciences, University of Cape Town, Rondebosch, South Africa

\section{Introduction}

12 Sutherland et al. (2015) developed a set of 10 priorities for biological recording, with a focus on monitoring biodiversity through citizen science. This paper develops an 11 th priority which, though not made explicit by Sutherland et al. (2015), was probably in mind. In the context of climate change, it is necessary to articulate the conscious and continuous need to keep biodiversity databases up-to-date; a corollary to this priority is the need for a metric to assess the concept of up-to-dateness. The objective of this paper is to meet this need to quantify up-to-dateness, and thus provide one measure of the temporal quality of a biological database.

21 The context of this paper is the ongoing collection of "atlas"-type data for a taxon, used to

22 generate on-demand, up-to-date distribution map for any species in that taxon. Such maps are

23 of fundamental value in establishing conservation priorities (Underhill \& Gibbons 2002,

24 Harrison et al. 2008). For example, the Second Southern African Bird Atlas Project began in 
252007 to serve as a five-year "snapshot" of bird distributions, as had been the objective of the initial bird atlas. Instead, the project remains ongoing in 2021, having morphed from the aim

27 of capturing a "snapshot" to recording a "video" of changing distributions (Harrison et al. 2008, Underhill 2016, Underhill et al. 2017). The key idea is that up-to-date distribution maps should be built on recent data. Representing the occurrence of a species at a location with an "old" record is unsatisfactory, and even misleading.

Therefore, the focus of this paper is to devise an algorithm which, in some way, measures the decay in temporal quality of biodiversity data. The algorithm attempts to answer the question: "how up-to-date is this collection of biodiversity records?". The algorithm is illustrated using the South African component of the Biodiversity and Development Institute's Virtual Museum database, which includes South Africa, Lesotho and eSwatini; however, the method is readily adapted to other contexts.

Methods

Algorithm

A biodiversity record is comprised of three components: species, place and date. The record

44 provides evidence that a particular species was recorded at the place on the date. The

45 algorithm proposed here requires each data point to be allocated to a "grid cell". For the

46 Virtual Museum, a 15-minute geographical grid is used, generating what are popularly known

47 as quarter-degree grid cells (even though there are 16 of them in a one-degree grid cell). For

48 the African Bird Atlas Project, a five-minute grid is in use, generating grid cells known as

49 pentads (Underhill 2016). North of c. $40^{\circ} \mathrm{N}$, geographical grid cells are no longer viable, 
50 because the east-west distance is far smaller than the north-south distance. The convention

51 then is to use grid cells measured in kilometres, as is done in the bird atlas projects of Europe

52 (e.g. Hagemeijer \& Blair 1997). In Britain, early projects made use of "counties" and "vice-

53 counties"; the proposed method works with any spatial units, provided they are standardized

54 through time.

55

For each record, the algorithm needs the three components: the date, the species, and the grid

cell to which it belongs. The algorithm itself has four steps:

1. For each grid cell, find the most recent date for each species;

2. Find the median of these dates, one per species (this date provides a measure of the temporal quality of the data for the grid cell);

3. Find the median dates for all grid cells in the region under consideration;

4. Calculate the median of the grid cell medians (this date, the median of the medians, is defined to be the temporal quality of the data in the region, i.e. up-to-dateness).

The algorithm is simple and transparent. At Step 2, the median date calculated for a grid cell

Step 4, the halfway point for all the grid cells in the region is calculated. The median dates of half of the grid cells fall before this date, and half fall later.

71 The algorithm can be applied not only to the current database, but also to historic databases created by only considering records submitted prior to a chosen date. The crucial statistic

73 then becomes the time interval, expressed in months or years, between the median of the

74 medians and the chosen date. If this interval is shortening through time, then the temporal 
75 quality of the database is improving, and vice versa. The trend in this statistic through time is

76 a powerful communication tool.

77

78

79

Application

80

The algorithm was applied to the databases of six of the projects within the Virtual Museum.

The Virtual Museum is a citizen science initiative which assembles photographic records for a series of taxa. It was originally developed for citizen scientists to contribute to reptile and

84 butterfly atlases in South Africa (Bates et al. 2014, Mecenero et al. 2013), and gradually expanded to cover a selection of other taxa. The databases for reptiles, butterflies, dragonflies and damselflies (Underhill et al. 2016), lacewings (Underhill et al. 2019), birds and frogs were evaluated. The algorithm used all records up to 22 June 2021. The "popular" names for the six databases are included in Table 1.

89

90 To illustrate the interpretation of the pattern in trends through time, the algorithm was also applied to dragonflies and damselflies records (OdonataMAP) on an annual basis over the decade since the project commenced in September (Underhill et al. 2016). To assess whether the rate of renewal of old records resulted in distribution maps becoming more or less up-to-

94 date through time, the algorithm was applied to observations uploaded by the ends of the six calendar years from 2015 to 2020.

96 


\section{Results}

Of the six Virtual Museum projects for which up-to-dateness was evaluated in June 2021, it is

101 clear that the distribution maps produced using the ReptileMAP data cannot be considered

102 "up-to-date". For half of the grid cells, the median date of the most recent record was 41

103 years prior to the date of the analysis. Similarly, the LacewingMAP database was 25 years

104 out of date, the FrogMAP database 21 years out of date, the LepiMAP database 12 years out

105 of date, and the OdonataMAP database 4.3 years out of date. The BirdPix database was

106 classified as 2.4 years, out of date (Table 1$)$.

107

108 Table 1. Summary statistics, including up-to-dateness, for six projects within the Virtual Museum, in

109 the 2,027 quarter degree grid cells in South Africa, Lesotho and eSwatini. Calculations were

110 performed on 25 June 2021, and the up-to-dateness is calculated as the difference between the median

111 date and 25 June 2021.

112

\begin{tabular}{|l|c|c|c|c|c|c|}
\hline Project & Grid & Species & Grid cell- & Records & Median date & $\begin{array}{c}\text { Up-to- } \\
\text { dateness } \\
\text { (years) }\end{array}$ \\
\hline BirdPix & 1,479 & 843 & 54,935 & 143,207 & 05 Jan 2019 & 2.4 \\
\hline FrogMAP & 1,763 & 132 & 15,253 & 50,545 & 17 Mar 2000 & 21 \\
\hline LacewingMAP & 837 & 408 & 5,918 & 11,259 & 20 Feb 1996 & 25 \\
\hline LepiMAP & 1,698 & 886 & 61,694 & 469,852 & 08 Jan 2009 & 12 \\
(butterflies only) & & & & & & 4.3 \\
\hline OdonataMAP & 1,126 & 162 & 17,437 & 108,842 & 09 Mar 2017 & \\
\hline ReptileMAP & 1,946 & 478 & 35,926 & 149,924 & 21 Sep 1980 & 41 \\
\hline
\end{tabular}


114 For OdonataMAP, the pattern of up-to-dateness reflects the typical behaviour anticipated as a

115 project evolves (Table 2). Over the first years to the end of 2013, coverage expanded rapidly,

116 and up-to-dateness remained constant. Thereafter, without an incentive to "refresh" records

117 of a species in a grid cell the up-to-dateness steadily moves backwards. Since 2016, a large

118 number of historical museum specimen records were included in the analysis, causing a 24-

119 month retrogression in up-to-dateness. In the subsequent four years from the end of 2016 to

120 the end of 2020, the up-to-dateness of the OdonataMAP database had slipped by four months,

121 from 46 months at the end of 2016 to 50 months at the end of 2020 (Table 2). At the end of

122 2020, for half of the 1098 grid cells visited, the median date of the most recent observation of

123 a species was after 10 November 2016, and before this date for the other half.

125 Table 2. Summary statistics, including up-to-dateness, for the OdonataMAP project of the Virtual

126 Museum in the 2,027 quarter degree grid cells of South Africa, Lesotho and eSwatini. These statistics

127 are calculated from the database using the records that had been uploaded by the end of each of the six

128 calendar years from 2011 to 2020.

\begin{tabular}{|l|l|l|l|l|l|l|}
\hline Period & Grid & Species & Grid cell- & Cumulative & Median date & Up-to- \\
& & & species & records & & dateness \\
(months)
\end{tabular}




\begin{tabular}{|l|l|l|l|l|l|l|}
\hline Up to 2019 & 1,062 & 162 & 15,739 & 82,497 & 14 Dec 2015 & 49 \\
\hline Up to 2020 & 1,098 & 162 & 16,556 & 98,505 & 10 Nov 2016 & 50 \\
\hline
\end{tabular}

131 Discussion

\section{Multiple measures of quality}

135 When applied to a biodiversity database, the term "data quality" is frequently used

137 word "quality" in describing the needs of biodiversity data in Europe, but never clearly

138 defined the term. There is a need to quantify the concept of "quality." In this context,

139 "quality" is a multi-dimensional concept; there is no single measure of quality.

140

141 Up-to-dateness, as presented here, is only one such measure of quality. It must be supported

142 by supplementary information which captures other dimensions of quality, i.e. the total

143 number of records within the region, the number of species represented, the number of grid

144 cells with records, and the number of grid cell-species records (the sum of the number of

145 species recorded in each grid cell). A further family of data quality refer to taxonomic issues:

146 the up-to-dateness of the taxonomy used for the database, and the percentage of records not

147 correctly identified to species level.

149 There are several qualitative dimensions implicit in the concept of biodiversity data quality.

150 The most frequently employed relates to gaps in coverage, i.e. spatial quality. Data are of

151 poor quality if they display gaps. These are either geographical gaps, areas for which little or 
152 no biodiversity data exists for an entire taxon; or gaps in the range map for an individual

153 species, i.e. places where the species probably occurs, but has not yet been recorded (false

154 negatives). A second dimension is yet another measure of temporal quality, which is

155 generally interpreted to mean that there are data for an extended time period, usually

156 measured in years or decades. In this context, biodiversity data for a region are said to have

157 poor temporal quality if they lack historical records, making it difficult to examine trends

158 through time.

159

160 For example, with data from 1,946 of 2,027 grid cells, ReptileMAP has the best spatial

161 coverage (96\%) of the six projects (Table 1). More nuanced measures of spatial quality

162 would need to 1) account of whether gaps in coverage tend to be in adjacent or scattered grid

163 cells, and 2) estimate the percentage of false negatives in the database. For 2), determining

164 the number which goes into the numerator when estimating this percentage is

165 straightforward; it is the sum of the numbers of species in each grid cell, 35,926 in the case of

166 ReptileMAP (Table 1). The denominator for the percentage requires an estimate of the

167 number of grid cells in the range of each species, a quantity which the atlas project aims to

168 determine. The number 35,926 is the total of the total number of grid cells in which the 478

169 reptile species have been recorded, and hence shaded in the distribution maps (Table 1).

171 Table 2 details improvements of other measures of quality in OdonataMAP data through

172 time. A set of distribution maps for 147 species made at the end of 2015 would have been

173 based on 14,994 records from 679 grid cells. In these maps, the total number of grid cells

174 which would have shown a species as present would have been 5,954 (Table 2). One year

175 later, after the inclusion of the historical data, maps for 159 species would have been based

176 on 36,900 records from 852 grid cells, and the total number of shaded grid cells would have 
177 almost doubled to 11,620 (Table 2). The improved spatial coverage was obtained by

178 sacrificing up-to-dateness (Table 2). By June 2021, the set of 162 maps including the

179 additional records collected from 2017 onwards would have had a total of 17,437 grid cells

180 showing a species as present (Table 1). This is an increase of 50\% since the end of 2016 ,

181 providing another useful measure of the improvement of the spatial quality of the

182 OdonataMAP database in South Africa, Lesotho and eSwatini in 4.5 years.

183

184 Thus quality, in this context, is a multidimensional concept. Temporal quality, as developed

185 here, is only one dimension in describing the value of the database.

186

An alternative approach to measure the temporal decay in quality

A biodiversity record provides evidence that a particular species was recorded at the place on the date. However, biodiversity literature seems to pay little or no attention to the reality that the value of a record steadily decreases as its date of occurrence recedes into the past. Aging records slowly but steadily become less and less valuable as evidence that a species still occurs at a given site.

In this paper, the concept of temporal quality of a biodiversity database is introduced, rooted

197 in the notion that data quality decays over time. However, the shape of the function which

198 describes the decay in value through time remains unknown, and needs to be quantified.

200 Here is an experimental approach to achieve this. Consider a substantially-sized sample (say $201 n=1000$ ) of correctly georeferenced biodiversity records with accurate dates. The sample 
needs to be stratified geographically and by date, possibly from one year ago to 100 years ago. Revisit the sites at which the records were made on the calendar date of the original record, and search for the species. Three outcomes are possible: (1) the species was recorded, and still occurs at the site, (2) there is still suitable habitat for the species, but it was not

206 found, and (3) the site has been transformed to such an extent that the species almost certainly no longer occurs. The data analysis should aim to develop a function which describes the average rate of decay in value of biodiversity records, i.e. to estimate the "halflife" of a record. It is likely that these decay functions vary between species. One objective,

210 probably unattainable, would be to establish a gold standard definition for the age at which a

211 record no longer provides suitable evidence that a species still occurs at a locality.

212 Distribution maps for a species could then exclude records older than this date, or plot them 213 differently to indicate regions in which the species has either actually gone locally extinct, or

214 where further search effort is needed to refresh evidence of its presence. Alternatively, 215 distribution maps could weight records by their age, so that older records have smaller 216 weights than newer ones.

218 The decay function also opens up new possibilities of developing a more nuanced measure of 219 up-to-dateness than the one developed here; for instance, using the last recorded date for each 220 species in a grid cell, one could apply the decay function to estimate the remaining evidential 221 value of the record, and calculate appropriate summary statistics.

Use of up-to-dateness to motivate citizen scientists

225 As demonstrated above for OdonataMAP (Table 2), the algorithm is not only applicable to

226 the current database. It can also be applied to generate trends from historical databases, 
created by only considering records submitted up to a certain point in time. When applied in this way, these trends may serve as motivational guidance for project leaders and citizen scientists.

230

231 For instance: "In this era of rapid development and climate change, any record of a species at 232 a locality which is more than two years old needs to be refreshed with a new one." The awareness of last recorded dates for the species in a grid cell is in itself a powerful motivational force for citizen scientists. There is a real challenge in seeking to detect the species that have the most-out-of-date last recorded dates in a grid cell.

237 The background to the six projects of Table 1 provides insights into the extent of their out-ofdateness. This information must be considered in communications with the citizen scientists participating in each project. Three of these projects (FrogMAP, LepiMAP and ReptileMAP)

240 are continuations of citizen science projects which produced published atlases for frogs

241 (Minter et al. 2004), butterflies (Mecenero et al. 2013) and reptiles (Bates et al. 2014)

242 respectively. In each case, the foundational data for the project were drawn from historical 243 databases in museums, other non-museum specimen collections, and literature. The explicit

244 objective of the citizen science fieldwork, which took place over five-year periods, was to fill 245 in distribution gaps with a goal of targeting false negatives, grid cells where the species likely 246 occurred but had not yet been recorded. This mentality of "filling in the gaps," has prevailed 247 in the continuation of these projects within the Virtual Museum. Thus, many observers do not 248 upload records to the Virtual Museum if the species has already been recorded in the grid 249 cell. The consequence is that the up-to-dateness of the databases slowly deteriorates.

250 Changing this outlook has proved challenging. 
252 OdonataMAP is a relatively new project (Underhill et al. 2016), and the cohort of citizen

253 scientists involved are relatively strongly attuned to the importance of repeated submission of

254 the same species from the same locality. To a large measure, this is because the project

255 includes a focus on generating data from which the phenology of adult dragonfly and

256 damselfly occurrence may be estimated. A by-product of this approach is that overall up-to-

257 dateness of the database deteriorated by only four months over four years (Table 2).

258

259 The foundational data for LacewingMAP were developed by Mervyn Mansell over a career;

260 they contain the global specimen database for the Neuroptera and Megaloptera, with records

261 dating back to the nineteenth century (Underhill et al. 2019). The citizen science database is

262 built on this platform. Contributions are opportunistic; no citizen scientist has a primary

263 interest in this taxon (Underhill et al. 2019). The rate of record submission is thus relatively

264 slow (but far faster than the rate of specimen acquisition in the decades when museum

265 collections were growing fastest (Underhill et al. 2019)). Thus, the fact that the database is 25

266 years out of date is not surprising (Table 2).

267

268 In contrast, although the BirdPix project of the Virtual Museum was started in 2012, active

269 promotion of the project commenced in 2017. By then a large number of grid cells had

270 received a small number of records each. Many citizen scientists have uploaded series of

271 historical photos taken in the early days of digital photography, or have even scanned and

272 submitted slides; this has effectively pushed the median of medians further into the past. For

273 the other projects within the Virtual Museum discussed here, most historical image

274 collections (for example of reptiles and butterflies) were uploaded during the formal atlas

275 periods for each taxon. However, in spite of these challenges, the BirdPix database was 2.4

276 years out of date in June 2021. 
278 The continuous presentation of up-to-dateness, preferably in graphical form, provides an

279 incentive to citizen scientists to keep the database up-to-date. This is an example of the

280 application of gamification to motivate data collection by citizen scientists (Ainsley \&

281 Underhill 2017). Gamification is the development of built-in strategies to encourage project

282 participation (in particular, it does not mean turning data-collection into a "game") (Ainsley

283 \& Underhill 2017).

284

285 For a citizen scientist to contribute towards bringing the median date for a grid cell closer to

286 the present, a straightforward strategy is to increase the number of species for that grid cell,

287 especially if it is still relatively easy to add species; each added species then has a current

288 date and moves the median for the grid cell forward. Similarly, to bring the median of

289 medians closer to the present would require undertaking fieldwork in grid cells which have

290 no data. This increases the number of grid cells with median dates; the new median dates are

291 current, and move the median of medians forward. Both strategies not only improve up-to-

292 dateness, they also positively impact other measures of completeness for the database (i.e.

293 spatial coverage).

294

\section{Local extinction}

297 Clearly, if a species goes extinct in a grid cell, its last recorded date can no longer be updated.

298 At some point, decisions about local extinction need to be made, so that a species can be

299 removed from the list over which the median for the grid cell is calculated. Mechanisms to do

300 this need to be devised, but will almost certainly be a combination of quantitative and

301 qualitative arguments. Species declared locally extinct need to remain on the species list, and 
302 flagged appropriately. The awareness among citizen scientists of local extinctions has the

303 potential to lead to civic awareness so that the impact of the project transcends science into

304 grassroots action and policy making (Loos et al. 2015).

305

Caveats to the measure of up-to-dateness

307

308 The measure of up-to-dateness described here is conditional on two factors: first, it only takes

309 account of grid cells for which data are available; and second, it only takes account of species

310 which have already been recorded in the grid cell. An absolute measure of up-to-dateness

311 would also account for the grid cells lacking data, and include an estimate of the number of

312 species in each grid cell.

313

314 The choice of the conditional measure was deliberate. Primarily, it is designed to measure the

315 up-to-dateness of the data already collected; other aspects of database quality can be

316 evaluated using other statistics, such as spatial coverage measures. Secondly, the choice of

317 conditional measure facilitates the use of gamification, as described above.

Acknowledgements

320

321 Itxaso Quintana, Magda Remisiewicz, Karis Daniel, Johan van Rooyen, Greg Distiller and

322 David Thomson made important comments on earlier drafts. The paper owes its existence to

323 the thousands of citizen scientists who have participated in the Virtual Museum. 


\section{References}

Ainsley J, Underhill LG. Gamification (persuasive design) in the Second Southern African Bird Atlas Project (SABAP2). Vogelwelt 2017;137:19-22.

Bates MF, Branch WR, Bauer AM, Burger M, Marais J, Alexander GJ, de Villiers MS, editors. 2014. Atlas and Red List of the reptiles of South Africa, Lesotho and Swaziland. Pretoria: South African National Biodiversity Institute; 2014.

Hagemeijer EJM, Blair MJ, editors. The EBCC atlas of European breeding birds: Their distribution and abundance. London: T \& A D Poyser; 1997.

Harrison JA, Underhill LG, Barnard P. The seminal legacy of the Southern African Bird Atlas Project. South African Journal of Science 2008;102:82-84.

342 Loos J, Horcea-Milcu AI, Kirkland P, Hartel T, Osváth-Ferencz M, Fischer J. Challenges for biodiversity monitoring using citizen science in transitioning social-ecological systems. Journal for Nature Conservation 2015;26:45-48.

346 Mecenero S, Ball JD, Edge DA, Hamer ML, Henning GA, Krüger M, et al., editors.

347 Conservation assessment of butterflies of South Africa, Lesotho and Swaziland: Red List and atlas. Johannesburg: Saftronics and Cape Town: Animal Demography Unit; 2013.

349 Minter LR, Burger M, Harrison JA, Braack HH, Bishop PJ, Kloepfer D, editors. Atlas and 
Washington, DC: Smithsonian Institution and Cape Town: Avian Demography Unit; 2004.

353

354 Sutherland WJ, Roy DB, Amano T. An agenda for the future of biological recording for 355 ecological monitoring and citizen science. Biological Journal of the Linnean Society 2015;115:779-784.

357

358 Underhill LG. The fundamentals of the SABAP2 protocol. Biodiversity Observations $2016 ; 7.42: 1-12$.

360

Underhill LG, Brooks M, Loftie-Eaton M. The Second Southern African Bird Atlas Project: protocol, process, product. Vogelwelt 2017;137:64-70.

Underhill LG, Gibbons D. Mapping and monitoring bird populations: their conservation uses. In: Norris K, Pain DJ, editors. Conserving bird biodiversity: general principles and their application. Cambridge: Cambridge University Press; 2002. p. 34-60.

Underhill LG, Navarro R, Mansell M. LacewingMAP: Progress report on the atlas of African Neuroptera and Megaloptera, 2014-2019. Biodiversity Observations 2019;10.10:1-21.

371 Underhill LG, Navarro R, Manson AD, Labuschagne JP, Tarboton WR. OdonataMAP:

372 progress report on the atlas of the dragonflies and damselflies of Africa, 2010-2016.

373 Biodiversity Observations 2016;7.47:1-10. 
bioRxiv preprint doi: https://doi.org/10.1101/2021.07.27.453997; this version posted July 27, 2021. The copyright holder for this preprint

(which was not certified by peer review) is the author/funder, who has granted bioRxiv a license to display the preprint in perpetuity. It is made available under aCC-BY 4.0 International license.

375 Wetzel FT, Bingham HC, Groom Q, Haase P, Kõljalg U, Kuhlmann M, et al. Unlocking

376 biodiversity data: Prioritization and filling the gaps in biodiversity observation data in

377 Europe. Biological Conservation 2018;221:78-85. 